\title{
The Set $R^{*}$ and Its Properties
}

\author{
Shengwu Zhu \\ Northwest University \\ No. 36 Xinmin street, Xincheng District Xi' an 710004, China \\ Tel: 86-29-8197-8655Ｅ-mail: zhufang04@1zu.cn
}

\begin{abstract}
In this paper, we shall give a set $R^{*}$ and indicate its properties, and thus, some abnormal results, such as the limit number may be successor, the natural number may be transfinite, the infinite set can not be equipotent to its proper subset etc., will be obtained.
\end{abstract}

Keywords: Remainder matrix, Axis number, Double-direction induction

1. On $R^{(n)}$

Assume the ordered set formed by front $(n+1)$ prime numbers on the number axis (We refer only to the nonnegative integers on right of number axis) in accordance with natural order be

$$
D_{n}=\left\{d_{0}, d_{1}, d_{2}, \cdots, d_{n}\right\}
$$

make product

$$
\mathfrak{Z}_{n}=\prod_{i=0}^{n} d_{i}
$$

, and denote the directed and closed segment from 0 to $\mathfrak{Z}_{n}$ on the axis by

$$
\bar{M}_{n}=\left[0-\mathfrak{Z}_{n}\right]
$$

The $\left(\mathfrak{Z}_{n}+1\right)$ integers in the $\bar{M}_{n}$ form an ordered set, in symbols

$$
Z_{n}=\left\{0,1,2, \cdots, \mathfrak{Z}_{n}\right\}
$$

For any $m \in Z_{n}$, dividing the $m$ successively by every prime in $D_{n}$, assume the remainders be respectively

$$
r_{m 0}, r_{m 1}, \cdots, r_{m n}
$$

and rewrite them as a single column matrix

$$
R^{(n)}(m)=\left[\begin{array}{c}
r_{m 0} \\
r_{m 1} \\
\vdots \\
r_{m n}
\end{array}\right]
$$

Obviously, for any one of numbers in $Z_{n}$ there is a definite matrix of single column corresponding with it. We arrange all these column matrices in natural order, such that they form a n-degree matrix of remainders

$$
R^{(n)}=\left[\begin{array}{cccc}
r_{00} & r_{10} & \cdots & r_{\mathfrak{Z}_{n} 0} \\
r_{01} & r_{11} & \cdots & r_{\mathfrak{Z}_{n} 1} \\
\vdots & \vdots & \ddots & \vdots \\
r_{0 n} & r_{1 n} & \cdots & r_{\mathfrak{Z}_{n} n}
\end{array}\right]
$$


So the integral points in $\bar{M}_{n}$, the integers in $Z_{n}$ and the columns in $R^{(n)}$ (respectively rejecting their last element) naturally form one-to-one correspondences (refer following 1.1, 1.2), each of them may therefore be replaced by the other.

The $R^{(n)}$ has following properties:

1.1 Any two different numbers in $Z_{n}$ except $\mathfrak{Z}_{n}\left(R^{(n)}\left(\mathfrak{Z}_{n}\right) \equiv R^{(n)}(0)\right)$ correspond to two different columns in $R^{(n)}$ (Two columns are called the same or equal when only when their corresponding elements at the same rows are all equal, in sign "”").

Proof: Assume that there were integers $i, j \in\left[0, \mathfrak{Z}_{n}\right)$ and $i<j$, such that $R^{(n)}(i) \equiv R^{(n)}(j)$, then by congruence property (Hua, 1964, P. 3, P. 22.), the difference $(j-i)$ can be integrally divided by each of all primes in $D_{n}$, therefore by $\mathfrak{Z}_{n}$ also. This is contrary to known that $(j-i)<\mathfrak{Z}_{n}$.

1.2 The all different columns in $R^{(n)}$ (Both the same first and last columns termed 0-columns which are composed all by zeros may disregard the last one) include all possible combinations taking respectively one remainder of each primes in $D_{n}$.

Proof: Assume $d_{i} \in D_{n}$, the all possible remainders of $d_{i}$ are

$$
P\left(d_{i}\right)=\left\{0,1,2, \cdots, d_{i}-1\right\}
$$

Taking $r_{i} \in P\left(d_{i}\right)(i=0,1,2, \cdots, n)$, we obtain a combination of remainders as $\left[\begin{array}{c}r_{0} \\ r_{1} \\ \vdots \\ r_{n}\end{array}\right]$. Evidently, the number of all possible such combinations is

$$
C_{d_{0}}^{1} \bullet C_{d_{1}}^{1} \bullet \cdots \bullet C_{d_{n}}^{1}=d_{0} d_{1} \cdots d_{n}=\mathfrak{Z}_{n}
$$

By 1.1 , there exactly are $\mathfrak{Z}_{n}$ different columns in $R^{(n)}$, and thus the 1.2 has been proved.

We may separate all the columns in $R^{(n)}$ into two classes: A column which does not contain the element zero is called a column of first class, otherwise a column of second class. The integral points in $\bar{M}_{n}$ (or numbers in $Z_{n}$ ) corresponding with the columns of first class are termed residual points (or residual numbers) of degree $n$, easily known

1.3 The number (total) of all columns of first class in $R^{(n)}$ is

$$
\mathfrak{Z}_{n}^{\prime}=\prod_{i=0}^{n}\left(d_{i}-1\right)
$$

. By 1.2 , we only except the element zero from $P\left(d_{i}\right)(i=0,1,2, \cdots, n)$, then get the combinations so done. Denote the ordered set (in natural order) of all residual numbers in $Z_{n}$ by

$$
\triangle^{(n)}=\left\{\delta_{0}^{(n)}, \delta_{1}^{(n)}, \cdots, \delta_{\mathcal{Z}_{n}^{\prime}-1}^{(n)}\right\}
$$

easily known, $\delta_{0}^{(n)}=1$, whereas the $\delta_{1}^{(n)}$ must be the next prime $d_{(n+1)}$. Obviously, the primes are infinite.

Calling the column $R^{(n)}\left(\frac{1}{2} \mathfrak{Z}_{n}\right)$ (the element at the first row is 1 , and the others all 0 ) the mid-column of $R^{(n)}$, we have

1.4 The two classes of columns in $R^{(n)}$ are all symmetrically distributive with reference to the mid-column. The mutually symmetric columns $R^{(n)}(m)$ and $R^{(n)}\left(\mathfrak{Z}_{n}-m\right)\left(m \in Z_{n}\right)$ satisfy

$$
r_{m i}+r_{\left(\mathfrak{Z}_{n}-m\right) i} \equiv 0 \bmod \left(d_{i}\right) \quad(i=0,1,2, \cdots, n)
$$

We call $R^{(n)}(m)$ and $R^{(n)}\left(\mathfrak{Z}_{n}-m\right)$ the mutually conjugate columns, in symbols, $\overline{R^{(n)}(m)}=R^{(n)}\left(\mathfrak{Z}_{n}-m\right)$, and also so do for two relative points or numbers. 
Two columns in $R^{(n)}$ are called mutually independent columns when only their corresponding elements of all the same rows are different, otherwise, mutually dependent columns. And so call the relative points or numbers.

1.5 For any integer $i \in Z_{n}$, when the $i$ is an odd the column $R^{(n)}(i)$ does not have any independent column of first class; When the $i$ is an even, then the $R^{(n)}(i)$ has such columns so many that the number $q$ satisfies

$$
\mathfrak{Z}_{n} \leq q \leq \mathfrak{Z}_{n}^{\prime} \quad \text { where } \mathfrak{Z}_{n}=\prod_{i=1}^{n}\left(d_{i}-2\right)
$$

1.6 For any even $h \in Z_{n}$, assume that its independent points of first class (i.e. independent residual points) laying within the interval $\left[h, \mathfrak{Z}_{n}\right]$ have number $q^{\prime}$, then in $\bar{M}_{n}$ the number of all pairs of residual points being $h$ apart (i.e. the distance between them is $h$ length units) is necessarily the $q^{\prime}$, and the converse is also true.

Proof: Assume that $\delta \in\left[h, \mathfrak{Z}_{n}\right]$ is a residual number independent of $h$, then the $R^{(n)}(\delta-h)$ must be a column of first class (i.e. the $(\delta-h)$ is also a residual number). If not, the element in $R^{(n)}(\delta)$ that corresponds with the element zero of $R^{(n)}(\delta-h)$ is necessarily equal to the relative element of $R^{(n)}(h)$. This contradicts that the $h$ and $\delta$ are mutually independent. Conversely, assume $\delta_{1}$ and $\delta_{2}$ be two residual numbers in $\bar{M}_{n}$ and $\delta_{2}-\delta_{1}=h$, then $\delta_{2} \in\left[h, \mathfrak{Z}_{n}\right]$, above all, the $\mathfrak{Z}_{2}$ must be independent of $h$. Because if not, for both $R^{(n)}\left(\delta_{2}\right)$ and $R^{(n)}(h)$, say, their elements of $\mathrm{i}$-th row were equal, then the element of i-th row in $R^{(n)}\left(\delta_{1}\right)$ must be zero. This contradicts that the $\delta_{1}$ is a residual number.

Particularly, in the $\bar{M}_{n}$, the number of all twin residual numbers is $\left(\mathfrak{Z}_{n}-1\right)$ pairs.

\section{On R}

According to congruence properties, the whole semi-axis of numbers forms infinitely many periodic segments: $\left[0, \mathfrak{Z}_{n}\right],\left[\mathfrak{Z}_{n}, 2 \mathfrak{Z}_{n}\right],\left[2 \mathfrak{Z}_{n}, 3 \mathfrak{Z}_{n}\right], \cdots$. Each of them corresponds to the same $R^{(n)}$.

Adding the next prime $d_{n+1}$ into the $D_{n}$, we obtain relatively $D_{n+1}, \mathfrak{Z}_{n+1}, Z_{n+1}, \bar{M}_{n+1}$ and $R^{(n+1)}$. Obviously, the $R^{(n+1)}$ involves $d_{n+1}$ ones of the same $R^{(n)}$ ranging periodically, but because of increasing a row of new elements at last (ranging the $P\left(d_{n+1}\right)$ periodically to $\mathfrak{Z}_{n}$ times), among the old columns of first class in $R^{(n+1)}$ there now are $\mathfrak{Z}_{n}^{\prime}$ ones to have translated into second class. Evidently, the columns of first class decrease relatively. For the sake of convenience, the process of translating from $R^{(n)}$ to $R^{(n+1)}$ through increasing the next prime is called regular evolution. Clearly, under the regular evolution, the properties stated before remain unchanged always.

Let $R^{(n)}$ by the order of natural numbers regularly evolves on infinitely (i.e. $n \rightarrow \infty$ ), then

$$
\begin{gathered}
D_{n} \rightarrow D=\left\{d_{0}, d_{1}, \cdots, d_{n}, \cdots\right\} \\
\mathfrak{Z}_{n} \rightarrow \mathfrak{Z}=\prod_{i=0}^{\infty} d_{i} \\
\bar{M}_{n} \rightarrow \bar{M}=[0-\mathfrak{Z}] \\
Z_{n} \rightarrow Z=\left\{0,1,2, \cdots, \mathfrak{Z}_{n}, \cdots, \mathfrak{Z}\right\} \\
R^{(n)} \rightarrow R=\left[\begin{array}{cccccc}
r_{00} & r_{10} & \cdots & r_{\mathfrak{Z}_{n} 0} & \cdots & r_{\mathfrak{Z} 0} \\
r_{01} & r_{11} & \cdots & r_{\mathfrak{Z}_{n} 1} & \cdots & r_{\mathfrak{Z} 1} \\
\vdots & \vdots & \cdots & \vdots & \cdots & \vdots \\
r_{0 n} & r_{1 n} & \cdots & r_{\mathfrak{Z}_{n} n} & \cdots & r_{\mathfrak{Z} n} \\
\vdots & \vdots & \cdots & \vdots & \cdots & \vdots
\end{array}\right]
\end{gathered}
$$

The $\mathrm{R}$ still preserves similar properties about mentioned before, above all

2.1 The $\mathrm{R}$ also possesses the last column $R(\mathfrak{Z})$ which is identical with the first column $R(0)$, both are composed by infinitely many zeros. Clearly, the proper factors of $\mathfrak{Z}$ involve every prime in $\mathrm{D}$, and thus $\mathfrak{Z} \equiv 0 \bmod \left(d_{i}\right)(i=$ $0,1,2, \cdots)$. Again, the index of each prime factor of $\mathfrak{Z}$ is degree 1 , hence the $\mathfrak{Z}$ is the minimal number by all primes as factors.

Similarly, the R possesses the mid-column $R\left(\frac{1}{2} \mathfrak{Z}\right)$, its element at the first row is 1 , and the others are all zeros. 
2.2 In $\mathrm{R}$, the first column $R(0)$ possesses the successor $R(1)=\left[\begin{array}{c}1 \\ 1 \\ \vdots \\ 1 \\ \vdots\end{array}\right]$, the last column $R(\mathfrak{Z})$ possesses the predecessor $R(\mathfrak{Z}-1)=\left[\begin{array}{c}d_{0}-1 \\ d_{1}-1 \\ \vdots \\ d_{n}-1 \\ \vdots\end{array}\right]$ (the $(\mathfrak{Z}-1)$ is therefore of definite meaning, and the $\mathfrak{Z}$ is a successor number), and the others possess both predecessor and successor. For this sake, we require only to subtract together 1 from every element of given column (if its some element, say one at mark $i$ row, is zero, then do after replacing it by corresponding prime $d_{i}$ ), or to add together 1 to every element of the column (if the element at mark $i$ row plus 1 is equal to $d_{i}$, then transform into zero).

Let $X=\left[\begin{array}{c}r_{0} \\ r_{1} \\ \vdots \\ r_{n} \\ \vdots\end{array}\right]\left(r_{i} \in P\left(d_{i}\right), i=0,1,2, \cdots\right), X_{k}=\left[\begin{array}{c}r_{0} \\ r_{1} \\ \vdots \\ r_{k}\end{array}\right]$, and $R_{k}$ be the submatrix formed by the front $(\mathrm{k}+1)$ rows of $\mathrm{R}$ (the $R_{k}$ is composed only by the $R^{(k)}$ and its periodic segments), then.

2.3 Any X is necessarily a column of R and any column of R must be some $\mathrm{X}$.

Proof: Assume that there were an $\mathrm{X}$ being not a column of $\mathrm{R}$, then there necessarily exists a certain $k \in \omega_{0}$ (the $\omega_{0}$ is ordered set of natural numbers), such that the $X_{k} \notin R_{k}$, and therefore $X_{k} \notin R^{(k)}$ as well as any periodic segment of $R^{(k)}$. This obviously contradicts the 1.2. Similarly, any column of R can only be some X.

2.4 In $\mathrm{R}$, any two columns but the $R(\mathfrak{Z})$ are mutually unlike.

Proof: (a) It may be alleged that, in R, there is no any other 0-column (its elements are all zeros) to be between the $R(0)$ and $R(\mathfrak{Z})$. Otherwise, if there were $\alpha(0<\alpha<\mathfrak{Z})$ such that $R(\alpha)$ could be a 0 -column, then $\alpha \equiv$ $0 \bmod \left(d_{i}\right)(i=0,1,2, \cdots)$, thus every prime in D would be proper factor of the $\alpha$, and so, $\alpha \geq \mathfrak{Z}$ (see 2.1), this is contrary to the assumption. (b) In R, there are no any two non-zero columns to be the same. Otherwise, if there were non-zero columns $R(\alpha)$ and $R(\beta)(\alpha, \beta \in Z$, and $\alpha<\beta)$, such that $R(\alpha) \equiv R(\beta)$, then by $R_{0-\alpha}$ denote the submatrix composed by all the columns from $R(0)$ to $R(\alpha)$ in $\mathrm{R}$, and on the $\mathrm{R}$ parallely translate it to right, so that to coincide with $R_{0-\beta}$ up to their last column, thus the two submatrixes, because their last columns are identical, by the double-direction induction (see late 3.2) easily known, the column (denoted by $R(\beta-\alpha)$ ) coinciding with the first column of $R_{0-\alpha}$ must be a 0 -column. By (a), this is impossible.

Denote the matrix obtained after to reject the last column $R(\mathfrak{Z})$ off the $\mathrm{R}$ by $R^{*}$, and conceive the $R^{*}$ as an ordered set of its columns in natural order, then the $R^{*}$ has minimal and maximal elements $R^{*}(0)$ and $R^{*}(\mathfrak{Z}-1)$ (called two extreme columns). Assume $\mathrm{X}$ be a non-extreme column of $R^{*}$, then clearly, the $\mathrm{X}$ has both immediate predecessor and successor. Therefore, the $R^{*}$ is a discrete set without any "limit (or inaccessible) element".

As $R^{(n)}$, the $R^{*}$ contains the columns of first class (e.g. in the column X let $r_{n}=n+1\left(n \in \omega_{0}\right)$ we get one), and in the $R^{*}$, each column has its conjugate one (the $R^{*}(0)$ and $R^{*}\left(\frac{1}{2} \mathfrak{Z}\right)$ are self-conjugate).

2.5 The potency (cardinal) of $R^{*}$ is equal to the potency of the Continuum (Xie, 1979, P. 2.): $\overline{\overline{R^{*}}}=c$. Proof: Denote the Continuum $[0,1]$ by $\mathrm{C}$, and let any infinite decimal $0 . m_{1} m_{2} \cdots m_{n} \cdots\left(0 \leq m_{i} \leq 9, i=1,2, \cdots\right)$ in 
C correspond to the element $\left[\begin{array}{c}0 \\ 0 \\ 0 \\ 0 \\ m_{1} \\ m_{2} \\ \vdots\end{array}\right]$ of $R^{*}$, we find that $\overline{\bar{C}}=c \leq \overline{\overline{R^{*}}}$. Next denote the set of all denumerable

sequences consisting of non-negative integers by $\mathrm{S}$, no doubt $\overline{\bar{S}}=c$, and $R^{*} \subset S$ when each column of $R^{*}$ is naturally conceived as such a sequence, and so $\overline{\overline{R^{*}}} \leq \overline{\bar{S}}=c$. Thus, $\overline{\overline{R^{*}}}=c$ (here followed the old view of cardinals and only make reference).

Use the $R^{(n) *}$ to express the ordered set of all columns of $R^{(n)}$ but the last one $R^{(n)}\left(\mathfrak{Z}_{n}\right)$, in definition, always $\mathfrak{Z}_{n}=R^{(n) *}$ for any $n \in \omega_{0}$, again when $n \rightarrow \infty$,

$$
\mathfrak{Z}_{n} \& R^{(n) *} \rightarrow \mathfrak{Z} \& R^{*}
$$

then $\mathfrak{Z}=R^{*}$ and thus $\overline{\mathfrak{Z}}=c$.

\section{On $R^{*}$}

Since the columns in $R^{*}$ and the integral points on number axis $\bar{M}$ (except the end point $\mathfrak{Z}$ ) form a one-to-one correspondence, thus we can directly use the columns of $R^{*}$ to label the integral numbers on $\bar{M}$ in the relation of correspondence (the end number $\mathfrak{Z}$ is labeled by $R^{*}$ itself). Obviously, these numbers not only include all natural numbers, but exceed them by far (e.g. the all residual numbers but the $R^{*}(1)$, the conjugate numbers of the natural numbers but zero etc. are all transfinite). Above all, in $R^{*}$ except $R^{*}(0)$, the all other numbers are successor ones, and any two consecutive numbers correspond to two points spaced out 1 apart on $\bar{M}$, and thus the $R^{*}$ (as well as the $\mathfrak{Z}$ ) may be regarded as extension of natural number set (such numbers as well as their points are still called integral ones).

Similarly to $R^{(n)}$, in $R^{*}$ the amount of all columns of first class is $\mathfrak{Z}^{\prime}=\prod_{i=0}^{\infty}\left(d_{i}-1\right)$. These numbers expressed by such columns except the $R^{*}(1)$ may be called generalized primes (or transfinite primes). Easily known, in $R^{*}$ the twin generalized primes are infinitely many pairs, their number is $(\mathfrak{Z}=1)$ pairs, where $\mathfrak{Z}=\prod_{i=1}^{\infty}\left(d_{i}-2\right)$. Of course, for any number in $R^{*}$, if its element of the first row is 0 , it is called an even; if 1 , an odd. In order to be clear about the orderity and connectivity of $R^{*}$, we have:

Axiom 1: The distribution of all integral points on $\bar{M}$ possesses: 1) equidistant property (all points are everywhere equispaced out by 1 length unit and, the extreme points have one-side consecutive points, the others have two-side ones); 2) increasing property (from left to right monotonically increasing); 3) completeness (single-linearly marked the all integers from 0 to $\mathfrak{Z}$ ). Such arrangement is termed the number axis order.

Axiom 2: Assume $\alpha, \beta(\alpha<\beta)$ being two integral points on $\bar{M}$, by $R_{\alpha-\beta}^{*}$ denote the subset of a segment from $R^{*}(\alpha)$ to $R^{*}(\beta)$ in $R^{*}$, let $\mathrm{U}$ and $\mathrm{V}$ be two nonempty and mutually complementary subsets of $R_{\alpha-\beta}^{*}$ (for any $R^{*}(x) \in R_{\alpha-\beta}^{*}$, either $R^{*}(x) \in U$ or $\left.R^{*}(x) \in V\right)$, then there exist $R^{*}\left(x_{1}\right) \in U$ and $R^{*}\left(x_{2}\right) \in V$, such that the $R^{*}\left(x_{1}\right)$ and $R^{*}\left(x_{2}\right)$ are consecutive.

\subsection{No doubt, the $R^{*}$ is an ordered set (with the number axis order).}

Note that, taking $R^{*}(\alpha) \in R^{*}$, the subset formed by all front columns of the $R^{*}(\alpha)$ is denoted by $A=\left\{R^{*}(0), R^{*}(1)\right.$, $\left.\cdots, R^{*}(\alpha-1)\right\}$ (when $\alpha=0, A=\varnothing$ ), particularly the $\mathrm{A}$ is called the pre-part of $R^{*}(\alpha)$ and signed as $R_{0-(\alpha-1)}^{*}$; the subset formed by all back columns of the $R^{*}(\alpha)$ by $B=\left\{R^{*}(\alpha+1), R^{*}(\alpha+2), \cdots, R^{*}(\mathfrak{Z}-1)\right\}$ (when $\alpha=\mathfrak{Z}-1, B=\varnothing$ ), then for any $R^{*}(x) \in R^{*}$, perhaps $R^{*}(x) \equiv R^{*}(\alpha)$, or $R^{*}(x) \in A$ (i.e. $R^{*}(x)<R^{*}(\alpha)$ ), or $R^{*}(x) \in B$ (i.e. $R^{*}(x)>R^{*}(\alpha)$ ). Obviously, it is impossible that $R^{*}(x) \in A$ and $R^{*}(x) \in B$ too (otherwise, there will be two identical columns in $\left.R^{*}\right)$.

3.2 Double-direction induction (DDI): By $R_{\alpha-\beta}^{*}$ denote the subset of a segment $\left(R^{*}(\alpha)<R^{*}(\beta)\right)$ in $R^{*}$, there is $R^{*}(\xi) \in R_{\alpha-\beta}^{*}$ which possesses property $\mathrm{Q}$, "for any column $R^{*}(x)$ of $R_{\alpha-\beta}^{*}$, assume the $R^{*}(x)$ possess the $\mathrm{Q}$, then its consecutive columns $\left(R^{*}(x-1)\right.$ and $\left.R^{*}(x+1)\right)$ also possess the Q", so every column of $R_{\alpha-\beta}^{*}$ possesses the 
Q.

Proof: Assume some columns of $R_{\alpha-\beta}^{*}$ do not possess the $\mathrm{Q}$, and all such columns form a set as $\mathrm{U}$, its complementary set be $\mathrm{V}$, then by axiom 2 , in $R_{\alpha-\beta}^{*}$ there exist consecutive columns $R^{*}\left(x_{1}\right) \in U$ and $R^{*}\left(x_{2}\right) \in V$, because the $R^{*}\left(x_{2}\right)$ has possessed the $\mathrm{Q}$, from assumption, the $R^{*}\left(x_{1}\right)$ possesses also the $\mathrm{Q}$, this is a contradiction.

3.3 The $R^{*}$ is not a well ordered set (see late 3.5). And thus, the $\mathfrak{Z}$ has been not an ordinal number (Thomas, 1978, P. 24-31.) in traditional meaning. Now define the numbers in $R^{*}$ and the $R^{*}$ itself (expressing the number $3)$ as axis numbers, then for each integral point on $\bar{M}$ there is a certain axis number corresponding with it. For consistence with $\mathfrak{Z}=R^{*}$ as ever, every axis number, as $R^{*}(\alpha) \in R^{*}$, may be defined as its pre-part, namely $R^{*}(\alpha)=R_{0-(\alpha-1)}^{*}($ when $\alpha=0$, its pre-part being $\varnothing)$.

This paper doesn't stipulate operations on axis numbers, only affirms the determinacy of predecessor and successor for given axis number. The $\mathfrak{Z}$ is an end number no its successor, so the $(\mathfrak{Z}+1)$ has not been axis number. As to $\mathfrak{Z}^{\prime}$ and $\mathfrak{Z}^{\prime}$, since all greater than 0 and smaller than $\mathfrak{Z}$, they must be axis numbers.

3.4 Each axis number (but zero) regarded as a set is not equipotent to its proper subset (PS).

Proof: Obviously, in $R^{*}$ any nonzero natural number has possessed such property. For convenience, assume that nonempty-subset $R_{0-\alpha}^{*}$ of $R^{*}$ is not equipotent to its any PS, we prove that $R_{0-\alpha_{1}}^{*}\left(\alpha_{1}=\alpha-1\right)$ and $R_{0-\alpha_{2}}^{*}\left(\alpha_{2}=\right.$ $\alpha+1$ ) are also not equipotent to their PS. 1). Suppose that the $R_{0-\alpha_{1}}^{*}$ were equipotent to its PS W, then add the element $R^{*}(\alpha)$ respectively to $R_{0-\alpha_{1}}^{*}$ and $\mathrm{W}$, and make it correspond to itself, so we find that the $R_{0-\alpha}^{*}$ and its PS $W \cup\left\{R^{*}(\alpha)\right\}$ are equipotent, this is contrary to assumption. 2) Suppose that the $R_{0-\alpha_{2}}^{*}$ were equipotent to its PS E, then a) If $R^{*}\left(\alpha_{2}\right) \notin E$, and $R^{*}\left(\alpha_{2}\right)$ corresponds to $R^{*}(x)$ of E, then reject both $R^{*}\left(\alpha_{2}\right)$ and $R^{*}(x)$ off $R_{0-\alpha_{2}}^{*}$ and E respectively, clearly $R^{*}(x) \in R_{0-\alpha}^{*}$, and so the $R_{0-\alpha}^{*}$ would be equipotent to its PS (E rejected the $R^{*}(x)$ ), being contrary to assumption. b) If $R^{*}\left(\alpha_{2}\right) \in E$, then in the corresponding relation of $R_{0-\alpha_{2}}^{*}$ and $\mathrm{E}$, either $R^{*}\left(\alpha_{2}\right)$ is oneself correspondence, in this case, reject the $R^{*}\left(\alpha_{2}\right)$ off $R_{0-\alpha_{2}}^{*}$ and $\mathrm{E}$, at once we find $R_{0-\alpha}^{*}$ being equipotent to its PS $E^{*}\left(\mathrm{E}\right.$ rejected the $\left.R^{*}\left(\alpha_{2}\right)\right)$, this is similar contradiction; or each $R^{*}\left(\alpha_{2}\right)$ corresponds to other element, to say, $R^{*}\left(x_{1}\right) \in E$ and $R^{*}\left(x_{2}\right) \in R_{0-\alpha_{2}}^{*}$ are the maps of $R^{*}\left(\alpha_{2}\right)$ from its set onto the opposite respectively, then reject the $R^{*}\left(\alpha_{2}\right)$ off each set, and make $R^{*}\left(x_{2}\right)$ correspond to $R^{*}\left(x_{1}\right)$, so we still find the $R_{0-\alpha}^{*}$ being equipotent to its PS $E^{*}$, and also obtain a contradiction to assumption. By DDI, the 3.4 has been proved. So the axis number is also cardinal like natural number.

3.5 Traditionally, the ordinal number $\omega_{0}$ defined by the ordered set of natural numbers is a "minimal transfinite number". In fact, the axis number system has shown that, between the finite and infinite there is no certain boundary and unbridgeable gulf. And so, so-called "minimal transfinite number" does not exist. Relatively, the "minimal transfinite cardinal number" does not exist too (old conclusion of infinite cardinals have been not enough to regard as criterion).

Usually, one stipulates the "natural numbers" being all finite. However, since each natural number itself also expresses the "number" of natural numbers (concretely, of all ones smaller than it), hence, if natural numbers are infinite many, then necessarily there will be some "infinite natural number" (generalized natural number). Since restricting of the decimal notation, when the order of units in a numeral changes into infinite, the traditional notation has had no method to express and to judge it, and so one counts it being no existing. However, when one has some new number scale and expressing means, the condition will be quite another.

\section{References}

Hua, Luogeng. An Introduction to the Theory of Numbers, Science publisher. P. 3, P. 22.

Thomas, Jech. Set Theory. London : Academic Press. P. 24-31.

Xie, Bangjie. Transfinite Number And Transfinite Theory-method, Jilin people publisher. P. 2. 